\title{
A Visual Approach to Efficient Analysis and Quantification of Ductile Iron and Reinforced Sprayed Concrete
}

\author{
Laura Fritz, Markus Hadwiger, Georg Geier, Gerhard Pittino, and M. Eduard Gröller
}
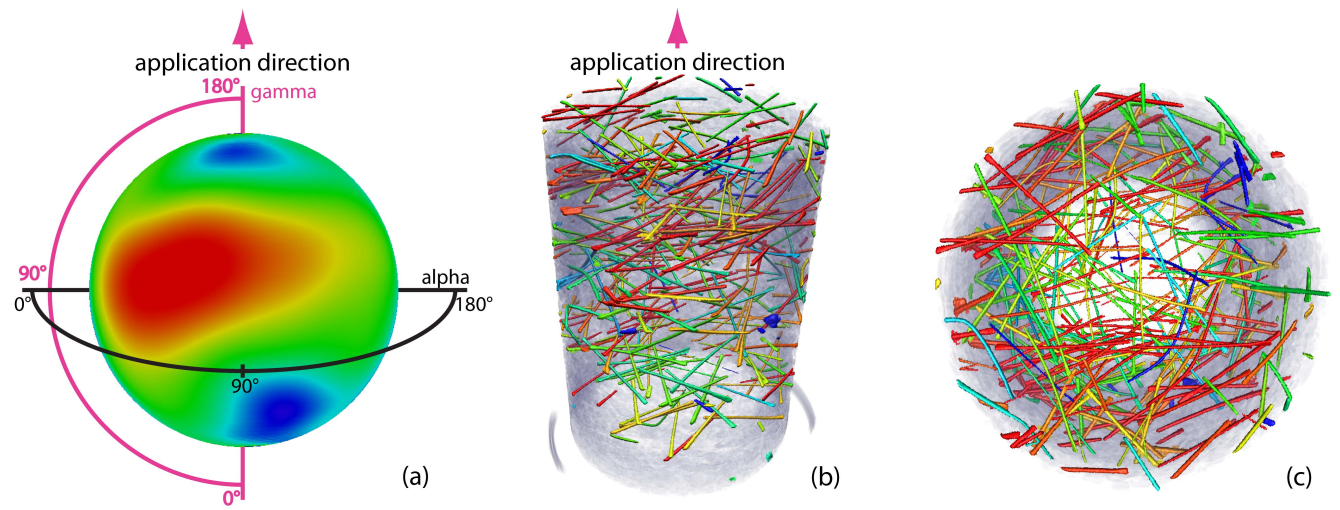

Fig. 1. CT-based visualization of drill cores from Steel Fibre Reinforced Sprayed Concrete (SFRSpC) enables inspection and analysis of the distribution of the steel fibres' directions, which is crucial for the material's mechanical properties. (a) Frequency of occurrence of directions color-coded in a Direction Sphere Histogram (red: highest, blue: lowest); (b) Frontal view; (c) Top-down view.

\begin{abstract}
This paper describes advanced volume visualization and quantification for applications in non-destructive testing (NDT), which results in novel and highly effective interactive workflows for NDT practitioners. We employ a visual approach to explore and quantify the features of interest, based on transfer functions in the parameter spaces of specific application scenarios. Examples are the orientations of fibres or the roundness of particles. The applicability and effectiveness of our approach is illustrated using two specific scenarios of high practical relevance. First, we discuss the analysis of Steel Fibre Reinforced Sprayed Concrete (SFRSpC). We investigate the orientations of the enclosed steel fibres and their distribution, depending on the concrete's application direction. This is a crucial step in assessing the material's behavior under mechanical stress, which is still in its infancy and therefore a hot topic in the building industry. The second application scenario is the designation of the microstructure of ductile cast irons with respect to the contained graphite. This corresponds to the requirements of the ISO standard 945-1, which deals with 2D metallographic samples. We illustrate how the necessary analysis steps can be carried out much more efficiently using our system for 3D volumes. Overall, we show that a visual approach with custom transfer functions in specific application domains offers significant benefits and has the potential of greatly improving and optimizing the workflows of domain scientists and engineers.
\end{abstract}

Index Terms-Non-Destructive Testing, Multi-Dimensional Transfer Functions, Direction Visualization, Volume Rendering.

\section{INTRODUCTION}

In recent years, the introduction and rapidly increasing use of $3 \mathrm{D}$ computed tomography in non-destructive testing (NDT) applications has opened up many powerful possibilities for analyzing and testing industrial parts such as cast metal without destroying them. However, many important tasks in this area such as designating the microstructure of ductile cast irons are still routinely carried out using a (destructive) metallographic approach and subsequent manual visual analysis. The metal under inspection is examined by sequentially removing layers via grinding or etching, and taking microscopic photographs. Due to the complexity of the observed structures, in current practice the clas-

- Laura Fritz and Markus Hadwiger are with the VRVis Research Center, Vienna, Austria, E-mail: \{laura|msh\}@vrvis.at.

- Georg Geier is with the Austrian Foundry Research Institute, Leoben, Austria, E-mail: georg.geier@ogi.at.

- Gerhard Pittino is with the Institute for Subsurface Engineering, University of Leoben, Austria, E-mail: Gerhard.Pittino@unileoben.ac.at.

- Meister Eduard Gröller is with the Vienna University of Technology, Vienna, AustriaE-mail: groeller@cg.tuwien.ac.at.

Manuscript received 31 March 2009; accepted 27 July 2009; posted online 11 October 2009; mailed on 5 October 2009.

For information on obtaining reprints of this article, please send email to:tvcg@computer.org. sification of important properties such as the form of graphite particles that are crucial for ductility is performed by human visual analysis and manual comparison with reference images. This process could be simplified tremendously by using computed tomography (CT) and powerful visual tools. However, currently there is a clear lack in visualization solutions that provide sufficient flexibility for exploration and inspection, as well as ultimately quantification. Moreover, the use of CT in non-destructive testing is increasingly employed for non-metallic or compound materials. For example, concrete is probably the most widely used non-natural material worldwide, and the use of CT for the inspection of concrete is becoming more widespread. However, the subsequent analyses are very time-consuming and in current practice still involve a considerable amount of manual work due to insufficient tools. In this paper, we describe novel visual workflows that are customized for specific applications with a high relevance for NDT practitioners. The presented approaches have been developed jointly with expert users from this domain, both from a more practical engineering and testing perspective, as well as from a materials research point of view. The first application scenario that we describe (Section 3 ) is the analysis of SFRSpC, illustrated in Figure 1, which is of very high importance in the building industry. We are concerned with the examination and analysis of the enclosed steel fibres with respect to their orientations and distribution relative to the application direction in which the concrete has been sprayed. SFRSpC is a relatively new material that is used in various applications in geotechnics. Although the dis- 


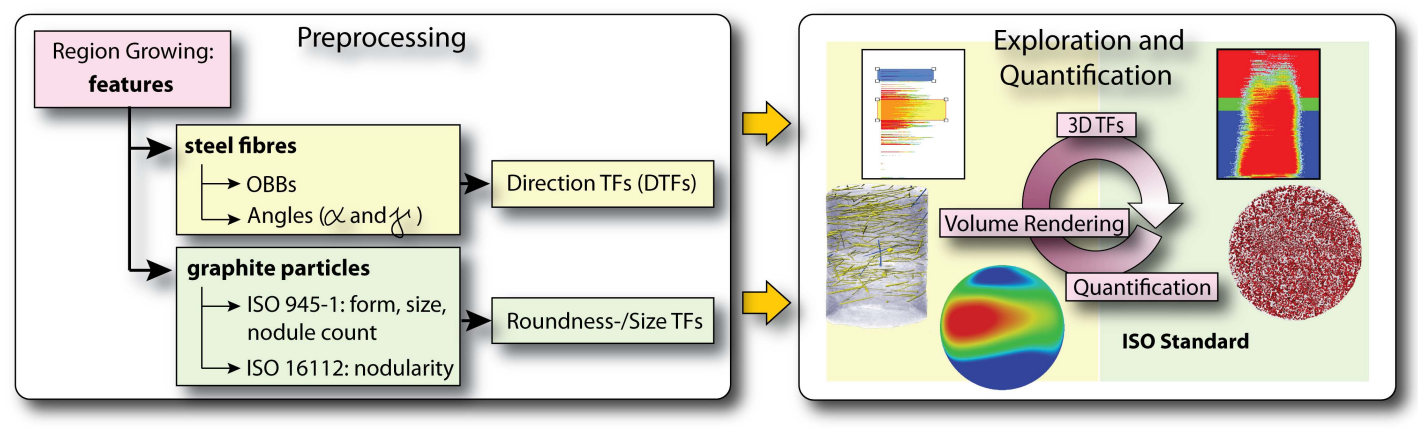

Fig. 2. Multi-pass region growing in a pre-processing step (left) results in detected features, which can then be explored and quantified interactively (right). The specific requirements of either application scenario are accommodated by pre-computing additional global as well as per-feature statistics for steel fibres or graphite microstructure, respectively. Default TFs for the exploration phase are also generated. The user explores features and feature classes by changing TFs, interactive exploration of statistical parameters, and the 3D volume view. The selected steel fibres are visualized in a Direction Sphere Histogram, whereas the graphite microstructure is classified according to ISO standard requirements.

tribution of fibre directions could strongly influence the mechanical properties of the material, they have not been properly explored yet. Instead, a continuous distribution was assumed. A preferred orientation of the fibres, however, can result from the application process of the concrete, which could significantly alter its mechanical behavior. Our solution provides an interactive exploration and automatic statistical analysis, based on automatic detection of the enclosed steel fibres, and the specification of transfer functions in the space of fibre directions. The second application scenario (Section 4) is the designation of the graphite microstructure in ductile cast irons, corresponding to the ISO standard 945-1. We illustrate how the analysis steps required by this established standard can be met much more efficiently by our system than the standard workflow of testing practitioners. We compute application-specific information such as histograms of particle diameter or roundness over density, which can then be explored interactively using transfer functions in these domains. This allows for interactive exploration and quantification in line with the requirements of the ISO standard. Transfer function presets enable easy classification according to whether the cast iron is vermicular, intermediate, or nodular, for example. Additionally, we compute properties such as the nodularity according to ISO standard 1611 .

The basis for the application-specific approaches in this paper is a pipeline for interactive exploration and quantification of industrial CT data [8]. This pipeline employs an interactive, visualization-driven approach for feature detection, exploration, and quantification. This is made possible by pre-computing a family of feature segmentations over parameter domains instead of fixed parameter settings. The precomputation has to be performed only once for a given data set. It is based on region growing that is computed in multiple passes, where the main region growing parameter such as the density variance allowed in a region is successively increased from pass to pass. The resulting features and their changes are tracked and stored in compact form in an additional feature volume and feature size curves [8]. Moreover, in contrast to the usual approach of segmenting only a single type of feature, in our system users can explore different feature classes simultaneously. In this paper, we extend this basic pipeline for the specific requirements of the two application scenarios outlined above through specialized transfer functions in feature space and additional visualization and quantification capabilities. Specifically, the novel application-triggered technical contributions of this paper are:

- The orientations of the steel fibres in SFRSpC are computed automatically in the pre-computation phase. Their distribution is visualized interactively in a Direction Sphere Histogram (DSH) that color-codes the frequency of occurrence of directions.

- Fibres are selected and visualized according to direction transfer functions (DTFs) in the domain of angles $\alpha$ and $\gamma$ relative to the application direction of the concrete, as well as material density. DTFs interactively determine the 2D and 3D visualization, as well as the set of fibres selected for depiction in the DSH.
- In addition to the size or volume of features, we have integrated the specific properties of feature diameter and feature roundness that are required by the ISO standards for classification of the graphite microstructure of ductile cast iron. These are used in application-specific transfer function domains, which enable the advantages of interactive exploration and quantification to be combined with the requirements of the respective standards.

- The classification of the microstructure of ductile cast irons is accomplished with respect to the contained graphite. This includes the nodularity and is done according to the requirements of the ISO 945-1 and ISO 16112 standards for single 2D slice images, as well as in $3 \mathrm{D}$.

Overall, we illustrate the large potential of customized, visual workflows to improve the daily routine of NDT practitioners, as well as opening up entirely new possibilities for exploration and analysis.

\section{Related Work}

We first review related work in the application domains used in this paper. The first international standard on graphite classification by visual analysis was adopted in 1975 as ISO 945:1975. It was left nearly unaltered until 2000, when work started on the first part of a revised version that was published as ISO 945-1:2008. The use of image analysis systems in many production laboratories of foundries that could lead to a more impartial classification of the graphite morphology required appropriate standards for their application. Currently, there is no information published when this part will become available. To evaluate the 3D shape of the graphite particles, metallographic serial sectioning techniques [17] can be used. For the special case of flake graphite, a 3D approach using focused ion beam (FIB) is used to investigate the whole network of spatially interconnected particles [20]. But as the sample is sectioned slice-wise, its integrity is lost. Furthermore, the data acquisition process is quite tedious. The resolution achieved with the FIB tomography is very high due to the imaging with scanning electron microscopy, but the acquired volume is quite small, especially with respect to the size of the graphite particles that are analyzed. The need for impartial classification and evaluation of the graphite microstructure has led to the development of several suggestions to analyze the form of graphite in metallographic sections $[11,16,19]$. These processes are based on different evaluation schemes such as image analytical parameters, shape parameters, or morphological transformations. However, fully automatic methods are very susceptible to the choice of parameters. In contrast to the case of ductile cast iron, the norm-guidelines for SFRSpC were evaluated by the European Committee for Standardization (CEN) in the last few years. This has resulted in general standards for sprayed concrete and steel fibres, and for their control [15]. These standardization efforts are under constant further development, where also national institutions such as concrete or construction societies are involved. We are not aware of any analysis of the influence of the fibre distribution in SFRSpC, except almost completely manual approaches [3]. 


\begin{tabular}{|l|c|c|c|}
\hline System features & MAVI [14] & VG [21] & Our system \\
\hline Region growing feature detection & $\mathrm{X}$ & $\mathrm{X}$ & $\mathrm{X}$ \\
Multi-pass region growing & - & - & $\mathrm{X}$ \\
Direction calculations in SFRSpC & $\mathrm{X}$ & - & $\mathrm{X}$ \\
Direction distribution visualization & - & - & $\mathrm{X}$ \\
Feature quantification & $\mathrm{X}$ & $\mathrm{X}$ & $\mathrm{X}$ \\
Feature ISO std quantification & - & - & $\mathrm{X}$ \\
\hline
\end{tabular}

Table 1. High-level comparison of the features of our system to two wide-spread existing systems: MAVI [14], and VGStudio MAX [21].

In visualization, the previous work most directly related to ours are size-based transfer functions [5], and specifying transfer functions over volume density, feature size, and region growing parameter [8]. The former computes a local size of the region surrounding any given voxel using scale space analysis. The latter detects connected regions and enables transfer functions over the size of these regions. We build on the second approach since it directly enables quantification of the features of interest, which is crucial in our application context. In our system, the detection of features such as steel fibres or graphite particles is performed using region growing [22]. The specification of seed points is crucial and can be tackled by evaluating multiple seed points at the same time [1], iteratively specifying seed points for region of interest selection [4], or allowing each voxel in the volume to potentially become a seed [8]. Gyulassi et al. [7] have presented two methods for the generation of topologically clean distance fields for the analysis of the filament structure of a porous material in molecular dynamics simulations. Using morse theory or an advancing front method they extract a stable core structure of the porous solid which is used for identifying material properties and comparing simulation results over different time steps. The two most related commercial tools that handle quantification of industrial CT data are MAVI [14], and VGStudio MAX [21]. Table 1 gives a high-level comparison of available features of these systems to the features of the system presented in this paper.

For volume visualization, we use GPU-based ray-casting [13, 18] with 3D textures and multi-dimensional transfer functions [12]. This allows for real-time rendering combined with considerable flexibility, including efficient memory managment for large volumes [2]. Region growing techniques have been integrated into volume visualization systems [9], which can also be used to define two-dimensional transfer functions that highlight the regions selected by region growing. This has also been demonstrated to be very useful in the context of industrial CT data [10]. However, the result is only an approximation and in general cannot reproduce the exact set of voxels of any given feature. In our context, it is crucial that all voxels are included in a feature to enable accurate quantification.

\section{Steel Fibre Reinforced Sprayed Concrete}

SFRSpC is a very important composite material that is used for various applications in geotechnics. It combines the high compressive strength of sprayed concrete with the strength after cracking through the addition of steel fibres. The material is brittle until a crack starts and becomes ductile through the steel fibres afterwards. Ideally, the steel fibres represent a continuous reinforcement if the fibres are randomly and homogeneously distributed. SFRSpC is used, for example, when a quick application of a shell is needed, e.g., when walls or rocks have to be secured, since then no steel mashes have to be applied before. SFRSpC can endure higher displacements of the rock due to the quasi-ductility of the material. Furthermore, the characteristics of the SFRSpC increase the safety for the workers, as cracks can be seen long before material failure.

\subsection{Application Scenario}

Although the mechanical properties of SFRSpC are generally a well studied problem, the steel fibres' orientation and distribution were disregarded in the past. Especially the orientation of the fibres has a great influence on the bearing capacity after the formation of cracks. The aim of this special study, realized at the Institute for Subsurface Engineering at the University of Leoben, is a statistical evaluation of the steel fibres' alignment in SFRSpC applied on a tunnel wall. The goal is to find out whether there is a prevailing characteristic orientation of the fibres that is due to the application direction of the sprayed concrete. Drill core samples are taken from the tunnel lining and are analyzed by means of industrial CT. In the previous, completely manual approach [3], the CT volume was loaded into a software tool where for each single steel fibre the coordinates of both ends had to be selected manually. For this, it was important that each steel fibre was processed sequentially in order to keep the storage order. Additionally, several formatting steps were required to finally get suitable coordinates for other tools to accomplish the statistical interpretation and direction visualization. The time needed for the individual required steps and the whole manual quantification process is summarized in Table 3. This process was very tedious and time-consuming, and required the use of several different software tools, which are also listed in Table 3. In contrast, our visualization-driven solution simplifies the steel fibre evaluation significantly, because it automatically computes and visualizes the directions of the fibres, and also provides different possibilities for statistical evaluation.

\subsection{Processing Pipeline}

The main stages of the processing pipeline are shown in Figure 2. The specific calculations required for this application are described in Section 3.2.2. They are performed in the automatic pre-processing phase, which is the basis for the interactive exploration and quantification described in Section 3.2.3.

\subsubsection{Data Acquisition}

The samples used in this section are core samples of a sprayed concrete shell of a tunnel. When drilling the cores, the axis of the cylindrical sample was aligned with the surface normal of the inner tunnel lining. This direction can be assumed to be the direction along which the sprayed concrete has been applied. It is mapped to the z-direction in our application. The data sets have been acquired from core samples using a microfocus x-ray CT-system scanned on a Phoenix $\mid x$-ray $v \mid$ tome $\mid x c$ of GE Sensing \& Inspection Technologies. For these samples, the resolution has to be high enough to correctly identify the steel fibres, which have a high x-ray absorption compared to the concrete matrix. A single fibre should be detected as one feature, and the detection of fibre fragments should be avoided because these can influence the detected orientations. We have investigated two drill cores in particular, denoted as SFRSpC 1 and SFRSpC 2, respectively. Figures 1 and 4 depict SFRSpC 1, and Figures 3 and 5 depict SFRSpC 2. See also Table 6.

\subsubsection{Pre-computation}

The computations necessary for the direction estimation of the steel fibres are performed during the region growing step in the preprocessing phase. After region growing, for each segmented feature an object oriented bounding box (OBB) is computed using principal component analysis (PCA). An OBB is a rectangular bounding box

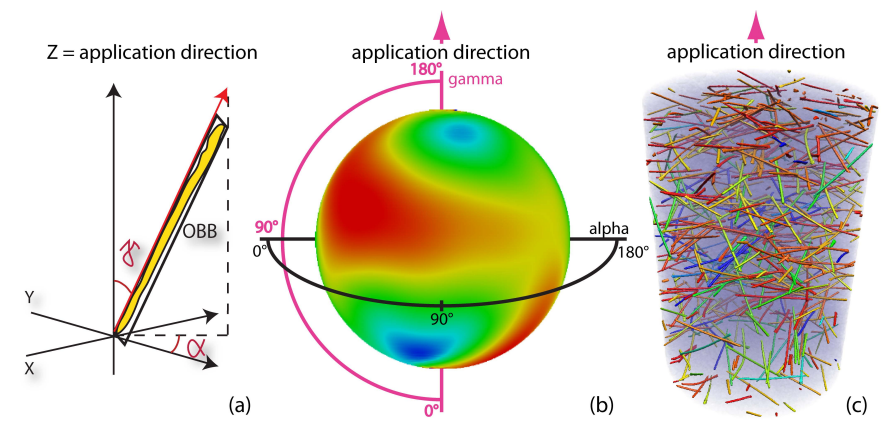

Fig. 3. Drill core SFRSpC 2 taken from the tunnel lining. (a) The longest axis of the OBB (black rectangle) enclosing the fibre (yellow) represents the feature's main direction. The angles $\alpha$ and $\gamma$ are required for the direction quantification of the steel fibres. (b) DSH of all fibres. (c) The color coding from the DSH can be used in the 3D volume view for inspection of fibres according to orientation. 

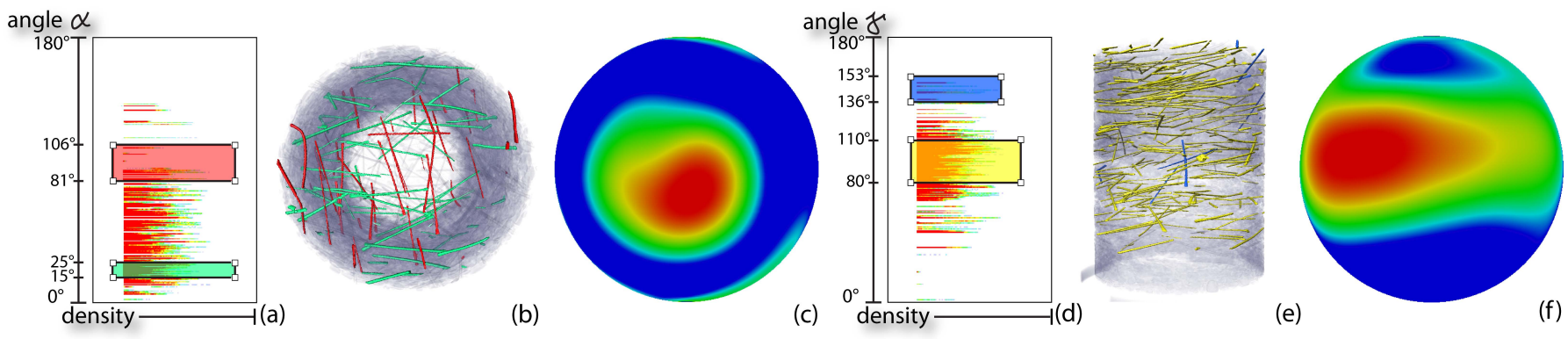

Fig. 4. The DTFs for $\alpha$ (a) and $\gamma(\mathrm{d})$, where the $\mathrm{x}$ axes show the density, and the $\mathrm{y}$ axes show the angle distribution of the steel fibres. For this application, it was sufficient to select the 2D histogram corresponding to a single suitable region growing parameter [8]. The 3D volume view immediately shows the fibres selected by widgets in the $\alpha$-DTF (b), or the $\gamma$-DTF (e), as well as their distributions in the DSHs, (c) and (f).

that approximates the arbitrary orientation of an object in 3D space [6]. PCA yields the three eigenvectors for the main axes, where the largest eigenvalue corresponds to the principal axis. The principal axis of the feature's OBB is used as direction vector in additional orientation calculations, as shown in Figure 3(a). To check the samples for preferred orientations of the contained fibres according to the requirements of the real-world analysis to be performed, we compute two main angles:

- Alpha $(\alpha)$ is the angle in the xy-plane normal to the concrete's application direction. It is in the range $\left[0^{\circ}, 180^{\circ}\right]$, since a halfcircle covers all orientations. See Figure 3.

- Gamma $(\gamma)$ is the angle between the $\mathrm{z}$-axis (the application direction of the sprayed concrete) and the feature direction in 3D. The range for $\gamma$ is $\left[0^{\circ}, 180^{\circ}\right]$ as well. See Figure 3 .

For each angle, a 3D histogram is created that corresponds to the distribution of angles, voxel densities, and region growing parameter. See Figure 4(a) for an example $\alpha$-density histogram, and Figure 4(d) for the corresponding $\gamma$-density histogram. In these figures, the horizontal axes show the density distribution, the vertical axis the angle distribution and the third dimension (not shown) corresponds to the change of the main region growing parameter such as variance [8]. The color coding indicates the number of voxels, where red indicates a high, and blue a low occurrence of feature voxels at a specific density and angle. These histograms are the domains of transfer functions for interactive exploration and quantification described below.

\subsubsection{Interactive Exploration and Quantification}

The distribution of directions of the steel fibres can be explored via DTFs in the domains of density and either $\alpha$ (Figure 4(a)) or $\gamma$ (Figure 4(d)), or both $\alpha$ and $\gamma$ (Figure 5(a)). For each desired feature class, a 2D TF widget can be specified interactively, which also specifies the color and opacity of the corresponding fibres in the $3 \mathrm{D}$ volume view (Figures 4(b), 4(e), and 5(b)). For each feature class, additional information like angular range, number of fibres, and the percentage of the volume covered by fibres of this class, is displayed and constantly updated. This enables quantification and guidance for exploration (Table 2). The $3 \mathrm{D}$ volume view allows one to inspect the current selection at any time, and determine if it is satisfactory. The mean and standard deviation of the selected features' angles are also calculated, and the
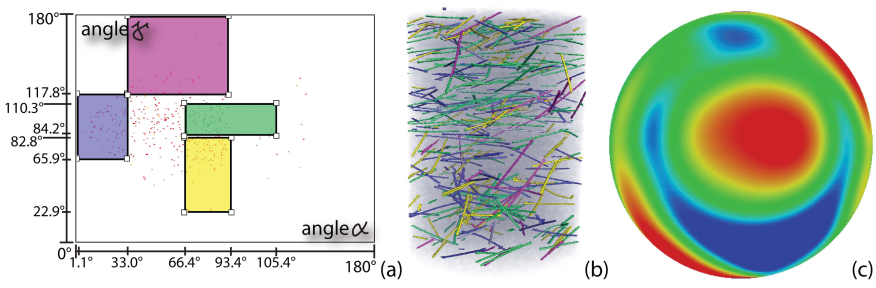

Fig. 5. (a) The DTF for $\alpha$ (x-axis) and $\gamma$ (y-axis). (b) The 3D volume view shows the selected steel fibres in the respective colors of the widgets, whereas (c) their direction distribution is visualized in the DSH. frequency distribution of the selected fibres' directions are color-coded in DSHs (Figures 4(c), 4(f), and 5(c)). These histograms depict the frequency of directions of the selected fibres, which is described in detail below. Figure 1(a) shows the DSH for all features in the SFRSpC 1 sample, where red color indicates a high frequency and blue color a low one. Because the two angles $\alpha$ and $\gamma$ are in the range $\left[0^{\circ}, 180^{\circ}\right]$, it is sufficient to plot the directions on a hemi-sphere. The mapping of the two angles $\alpha$ and $\gamma$ onto the hemi-sphere, relative to the application direction of the sprayed concrete, is illustrated in Figures 1(a) and 3(b). The color coding of the DSH can also be used in the 3D volume view, where each fibre is assigned a color corresponding to the number of fibres in the same direction (Figures 1(b) and (c)).

\subsubsection{Direction Sphere Histogram (DSH) Computation}

For the DSHs displayed by our system and depicted in Figures 1(a), 3(b), 4(c,f), and 5(c) we use a tessellated sphere as the domain. Each vertex of the tessellation comprises a histogram bin with a corresponding floating point count that can easily be mapped to a color using a simple 1D color table. The spheres are rendered using OpenGL, where the colors are mapped to vertex colors and interpolation is used within each triangle. For each fibre selected by means of a DTF, its direction must be counted in the DSH. In order to generate smooth histograms, for each fibre we splat a Gaussian kernel centered at the fibre's direction onto the spherical domain. The histogram count of every vertex that is covered by a (truncated) Gaussian splat kernel is incremented according to the spherical distance of the vertex to the center of the Gaussian, i.e., the fibre's original direction. This distributes the fibre's direction smoothly in a small neighborhood of bins around it. Since the integral of the Gaussian kernel is 1.0, this results in a good tradeoff between accuracy and smooth histogram appearance.

For efficient run-time computation of DSHs, we pre-compute a sampling of the weights of a Gaussian distribution with a user-defined standard deviation $\sigma$ that controls the size of the splat. For the full size of the truncated Gaussian kernel, we use a radius of $4 \sigma$. For simple implementation, the function values according to the distance from the center of the Gaussian kernel are stored in a 1D gauss_array. This is shown in Figure 6(b), and used during splatting. While tessellating the sphere, the angular distances and number of steps between two successive longitudes or latitudes, respectively, of the tessellation are stored in a sphere_distance_array. This enables fast computation of the area on the sphere that is covered by a Gaussian of a given size.

To calculate the values for the histogram bins, the contributions of the directions of all selected fibres are summed up. All fibres' main directions are retrieved from the previously calculated OBBs, and the

\begin{tabular}{|c|c|r|r|}
\hline angle & range & feature count & \% of part vol. \\
\hline$\alpha$ & $15^{\circ}-25^{\circ}$ & 53 & 0.14 \\
Fig. $4(\mathrm{a}, \mathrm{b}, \mathrm{c})$ & $81^{\circ}-106^{\circ}$ & 37 & 0.08 \\
\hline$\gamma$ & $80^{\circ}-110^{\circ}$ & 234 & 0.60 \\
Fig. 4 (d,e,f) & $136^{\circ}-153^{\circ}$ & 9 & 0.02 \\
\hline
\end{tabular}

Table 2. Quantification of the steel fibres in SFRSpC 1 selected by the classification widgets shown in Figure 4. 

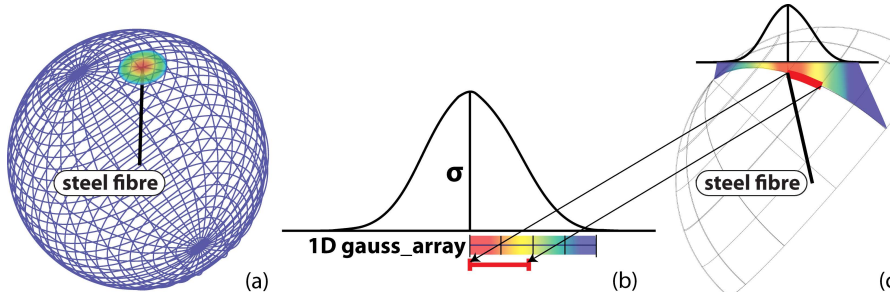

(a)
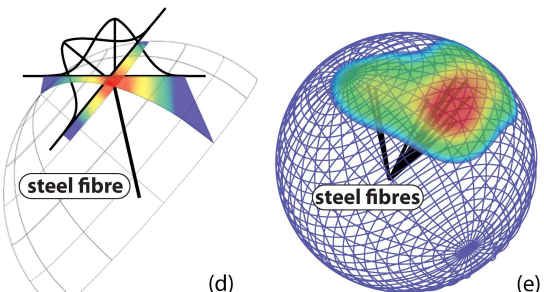

(e)

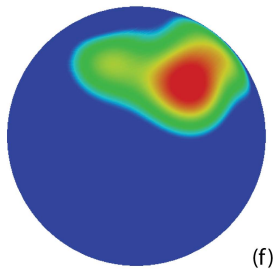

(f)

Fig. 6. The domain of a DSH is a sphere (or hemi-sphere) tessellated using a simple latitude/longitude tessellation. The directions of steel fibres are counted in the histogram by splatting a Gaussian kernel centered at each direction vector onto the sphere's vertices (a). This is simplified by using a 1D look-up table for one half of a Gaussian (b), which is accessed twice to obtain a 2D Gaussian distribution via tensor product (c,d). At each vertex, the contribution of the surrounding fibres is summed up, which results in the corresponding color distributions on the DSH (e,f).

vertices covered by the truncated Gaussian centered at each direction are computed. The histogram bin counts are then updated according to their distance to the center of the Gaussian (Figure 6(c,d)). Figure 6(a) shows this procedure for one fibre, whose Gaussian splat covers a certain area in longitude and latitude on the sphere. The sum of multiple fibres yields the corresponding distribution in the DSH as shown in Figure 6(e). Finally, the histogram values on the entire sphere are mapped to colors (Figure 6(f)). This technique ensures a fast recalculation of the DSH when the selection of fibres has changed. Only the counts at the vertices have to be computed again, using simple look-ups in the gauss_array according to the distance looked up in the sphere_distance_array. Full re-computation is only required when the size/standard deviation $\sigma$ of the Gaussian splats is changed by the user.

\subsection{Discussion and Results}

The final quantification of steel fibre directions is often needed for all fibres in the volume, for which a single classification widget in any of the possible DTF domains is sufficient. For example, in the direction quantifications depicted in Figures 1 and 3, a single classification widget was used to mark all steel fibres in the volume. Even in this case interactively moving and changing the widget in any of the DTFs allows users to quickly and effectively gain an overview and understanding of the fibre distribution in the volume, while inspecting various angular ranges of interest. However, assigning different angular ranges to different feature classes by using multiple classification widgets allows for a much more fine-grained analysis. An example of such a selective classification is shown in Figure 4. There, only a subset of the steel fibres has been selected by the user via classification widgets, resulting in the quantification of individual feature classes given in Table 2.

For practical evaluation of our system we have made a comparison with the results generated by the completely manual approach performed at the Institute for Subsurface Engineering at the University of Leoben [3]. The result of our system was exactly the same as the manual approach. However, it could be achieved in a fraction of the time, as illustrated in Table 3. It also provided the domain experts with many additional possibilities for real-time exploration of the data set. The distributions in the $\gamma$-DTF (Figure 4(d)) as well as in the DSH (Figure 1(a)), clearly show that there occurs a high fibre concentration at about $\gamma=90^{\circ}$, which is close to a right angle with respect to the concrete's application direction. Also for the $\alpha$-direction, the DTF (Figure 4(a)) and the DSH (Figure 1(a)) show a preferred orientation between $\alpha=10^{\circ}$ and $\alpha=90^{\circ}$. Our expert collaborators assume that the application direction in reality was not exactly orthogonal to the tunnel lining, which has led to the observed orientation according to $\alpha$. As was often tested by using flexural tension tests, SFRSpC keeps a part of its strength after the building of cracks because the steel fibres

\begin{tabular}{|l|c|c|}
\hline processing steps & manual & automatic \\
\hline Automatic pre-computation & - & $1.7 \mathrm{~min}$ \\
Selection of steel fibres & $30 \mathrm{~h}$ (View Expert) & $5 \mathrm{sec}$ \\
Statistical analysis & $20 \mathrm{~h}$ (Microsoft Excel) & immediate \\
Direction visualization & $5 \mathrm{~min}$ (Stereo 32) & immediate \\
\hline Total time for vis. and quant. & $\sim 50 \mathrm{~h}$ & $<2 \mathrm{~min}$ \\
\hline
\end{tabular}

Table 3. Times for the manual approach (tools in parentheses) vs. our automatic method for direction analysis of steel fibres in SFRSpC. aligned orthogonal to the crack interact against the bending direction. Due to the observed preferred orientation of the fibres, its effectiveness increases if they lie parallel to the surface, and decreases if they lie parallel to the spraying direction, i.e., normal to the surface. A direction visualization of the second drill core sample (SFRSpC 2) taken from the same tunnel lining, including more steel fibres, is shown in Figure 3. This analysis emphasizes the observations described above. The guidelines (EFNARC European Specification for Sprayed Concrete 1996) and standards (EN 14488-7) for sprayed concrete currently only provide the examination of the fibre content in fresh or already hardened probes. In fresh concrete, the fibres must be washed out. In already hardened concrete, the fibres have to be mechanically quarried out of the concrete and cleaned afterwards. Only then the number of fibres in the sample can be estimated. With our system, it is now possible to estimate not only the number of fibres, but also their orientation in the sample at hand. Because the fibres' orientation has a significant impact on the mechanical behavior of SFRSpC, our domain expert collaborators think that in general the quantification of SFRSpC and the analyses of the related properties can be improved tremendously in the future by using such a system, as well as enabling new scientific discoveries in this engineering discipline.

\section{Ductile Cast Iron}

In ductile iron, the majority of the carbon is present as graphite spheres in the microstructure, which exhibits higher strength and ductility than for lamellar shaped graphite particles. The main share of ductile cast iron is used in the production of pressure pipes, followed by automotive parts and different parts for mechanical engineering. To achieve desired material properties, an appropriate microstructure has to be ensured during production. One major influence is the formation of the graphite. Ideally, the microstructure consists of small, finely dispersed spheres of graphite all over the casting which provides the highest strength and ductility as well as high fatigue strength at the same time. Due to different production constraints, this cannot be achieved in every casting or even in every part of the casting. For this reason, the examination of the graphite formation is often required in quality specifications. To harmonize these examinations, the standard ISO 945-1 (ICS: 77.080.10) was established. The classification of the graphite's microstructure in cast irons is based on a visual comparison. In quality specifications for castings, minimum requirements concerning graphite form, particle density, and maximum particle size are common in the casting industry.

\subsection{Application Scenario}

During the designation of microstructure in cast irons according to the requirements of the standard ISO 945-1, the metallographic samples of ductile cast iron materials are examined under a microscope. The graphite inclusions are classified by its (1) form, (2) distribution, and (3) size. The graphite distribution designation is only specified for a form that is not allowed to occur in ductile cast iron, and is therefore ignored in our process. The standard ISO 945-1 provides reference images of schematic microstructures of cast iron as a basis for classification. Form and size of the graphite inclusions observed on the sample's surface are determined via comparison with the reference images that resemble them most closely. However, depending 


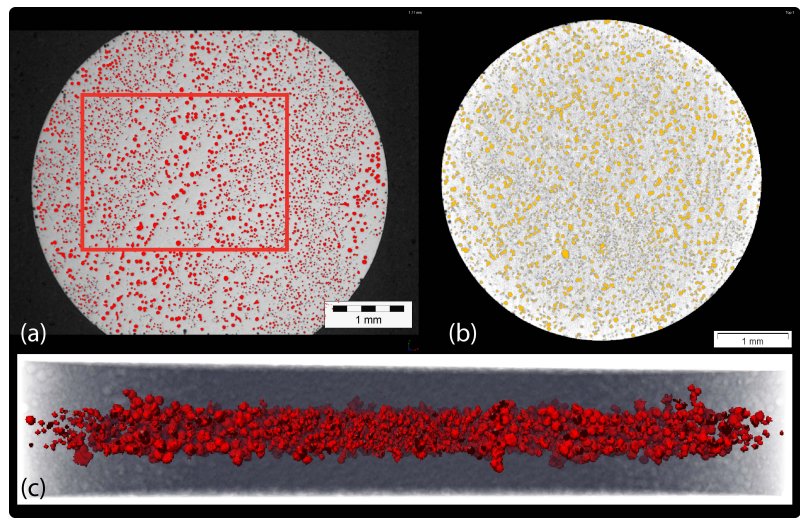

Fig. 7. The quantification of ductile cast iron according to ISO 945-1 and ISO 16112 is currently accomplished on the basis of 2D microscopic slice images (a), where a representative section is selected for quantification (red frame). For evaluation, we also compare CT results in $2 \mathrm{D}$ with a segmentation obtained via VGStudio MAX [21] on the same data (b). Our system is also able to perform quantification fully in 3D (c).

on the location where the metallographic samples were taken, the results can show a significant variation. Additionally, the preparation of the sample itself is very important. Careful grinding and polishing of the sample is crucial. The graphite particles should appear in their original form and size, so that an unacceptable alteration of the microstructure is avoided. The whole conventional quantification process is limited to 2D, where the comparison of the actual microstructures with the schematic images depends on the subjective impression of the metallographer. Our 3D approach via CT-scanning and interactive visualization-driven exploration of the whole data set, can overcome the drawbacks of the conventional, manual approach. A higher number of individual particles are taken into account, and scattered graphite particle deviations can be found more easily. Additionally, our 3D approach should help to resolve the statistical aberrations occurring when calculating the form factor of an inherently 3D graphite inclusion by means of a single $2 \mathrm{D}$ slice image. By automating the quantification step, the results are no longer constrained to the metallographers' subjectivity.

\subsection{Processing Pipeline}

An overview of the individual stages of the processing pipeline for the quantification of ductile cast iron is shown in Figure 2.

\subsubsection{Data Acquisition}

The sample presented in this section was taken from a thin walled casting from serial production, which had to be checked for its casting quality. The data have been acquired from material samples using a microfocus X-ray CT system. The resolution must be chosen high enough to ensure that the graphite particles can be represented by several voxels in the reconstructed volume. The accuracy of the analysis also depends on the average number of voxels per graphite particle.

\subsubsection{Pre-computation}

The parameters required for graphite microstructure quantification are computed in the pre-computation phase during region growing. The relevant indications for quantifying the graphite spheres according to the ISO 945-1 standard are their size, form, and nodule count. These are measured by means of the smallest sphere enclosing a particle. In the ISO standard, the form of each graphite particle is denoted by the roundness of the particle. The nodularity is calculated as a parameter of the dominant form in the microstructure, according to the ISO standard 16112, from the percentages of the different graphite forms in the sample. For each particle, we estimate the smallest enclosing bounding sphere. In this context, the size of a particle is defined as the diameter of its enclosing sphere. Figure 8(d) describes the sizes according to the classes defined in ISO 945-1. To estimate the form factor for a given graphite particle, we have to calculate its roundness, which is computed in $3 \mathrm{D}$ as follows:

$$
\text { Roundness }=\frac{3 V_{\text {particle }}}{4 r^{3} \pi},
$$

where $V_{\text {particle }}$ is the volume of the particle, and $r$ is the radius of the smallest enclosing bounding sphere. The roundness therefore is the ratio between the volume of the particle and the volume of the enclosing sphere. According to this property, the particles are classified as belonging to one of three relevant (of six overall) different forms, representing the typical types of graphite particles in such data:

- vermicular (form factor III): $0.0 \leq$ Roundness $<0.525$.

- intermediate (form factor V): $0.525 \leq$ Roundness $<0.625$.

- nodular (form factor VI): $0.625 \leq$ Roundness $<1.0$.

The nodularity, regarding the three different morphology groups as defined in accordance to ISO 16112, is calculated as follows:

$$
\operatorname{Nod}[\%]=100 \frac{\sum V_{\text {nodular }}+0.5 \sum V_{\text {intermediate }}}{\sum V_{\text {nodular }}+\sum V_{\text {intermediate }}+\sum V_{\text {vermicular }}},
$$

where $\sum V_{\text {nodular }}, \sum V_{\text {intermediate }}$, and $\sum V_{\text {vermicular }}$ are the number of voxels of all particles with roundness factor nodular, intermediate, and vermicular, respectively. To estimate the nodule count, only the particles of form factor V and VI (see Figure 8(c)) per $\mathrm{mm}^{3}$ are of relevance. We extend the planimetric method to the $3 \mathrm{D}$ domain, such that we compute the nodule count $n$ as $n=N / V_{\text {ref }}$ where $N$ is the number of nodules (i.e., particles classified as forms V and VI) counted in a reference subset of the whole volume chosen for measuring the nodule count, and $V_{\text {ref }}$ is the volume of this subset.

To enable interactive exploration and quantification, according to the parameters relevant for ISO 945-1 and ISO 16112 described above, we design two new 3D TFs. Graphite particles, are classified by means of the 3D domains of density, feature diameter or feature roundness, and the main region growing parameter [8]. The 2D TFs of a given region growing parameter are shown in Figures 8(a) and (d). The number of features is color-coded, where red corresponds to a high, and blue to a low occurrence of features at the current position. The backgrounds of the histograms are color-coded according to the graphite's size or roundness classes, as defined by the ISO standards.

\subsubsection{Interactive Exploration and Quantification}

During exploration, the current classification is shown in real-time in a $3 \mathrm{D}$ volume view and three orthogonal slice views. In order to explore and classify feature classes, the corresponding features are mapped to color and opacity, so that the user can observe and control the selections made. The desired quantification of the ductile cast iron according to the form parameter of the ISO standard 945-1 is specified via the (density, feature roundness) TF shown in Figure 8(a). The formdistribution of the graphite's microstructure is explored via the quantification widgets, where the selection of the graphite particles is constantly updated in the $3 \mathrm{D}$ volume view. As already explained for the SFRSpC, additional parameters like the roundness- and density range, or the nodularity of the features, selected by the quantification widget, are constantly displayed to help guide exploration. Our system provides automatic color coding according to the form factors defined in the ISO standard. Figure 8(a) shows a (density, feature roundness) histogram. The feature's roundness is shown on the vertical axis, where the roundness increases from bottom to top in the range $[0.0 ; 1.0]$. The user can select different feature classes, which are colored corresponding to the widget colors and opacity. If desired, the form factor's color coding can be transferred to the 3D volume view to obtain a better overall impression of the data, as shown in Figures 8(b) and (c). In contrast to SFRSpC, the quantification of individual form-groups may be of interest. In this case, different widgets are placed to mark the desired areas, and they are quantified separately. For example, to estimate the nodule count only features of form factor $V$ and $V I$ are of interest and can therefore be selected individually (see Figure 8(c)). The same procedure, using the (density, feature diameter) TF, is performed to estimate the size-distribution of the graphite particles, as 

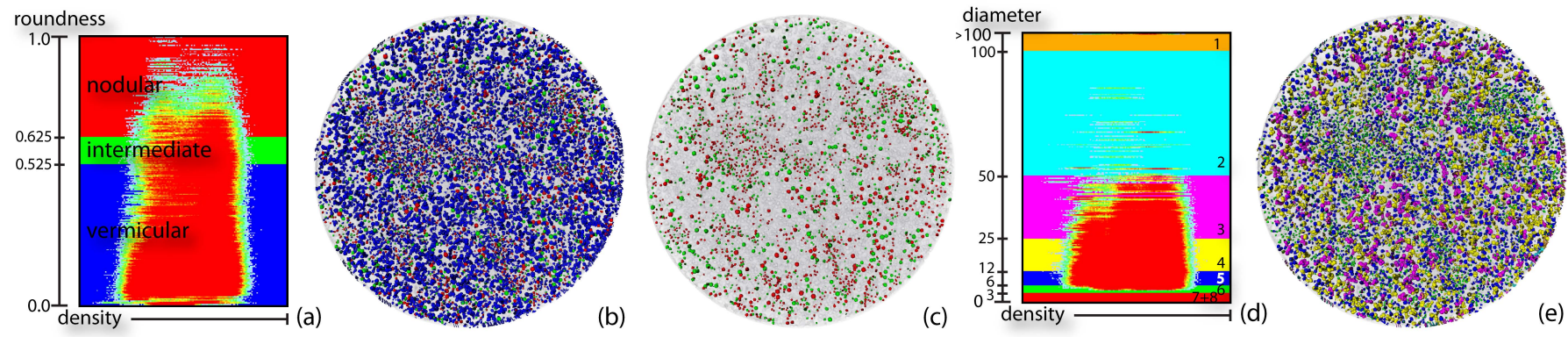

Fig. 8. To quantify the graphite microstructure according to the form and size parameters of the ISO 945-1 standard, one appropriate region growing parameter is selected interactively: (a) and (d). The color coding according to the three form factors (b) and the eight size-classes (e) is also applied in the 3D volume view. For the nodule count, only the graphite microstructure of forms $V I$ and $V$ are taken into account (c).
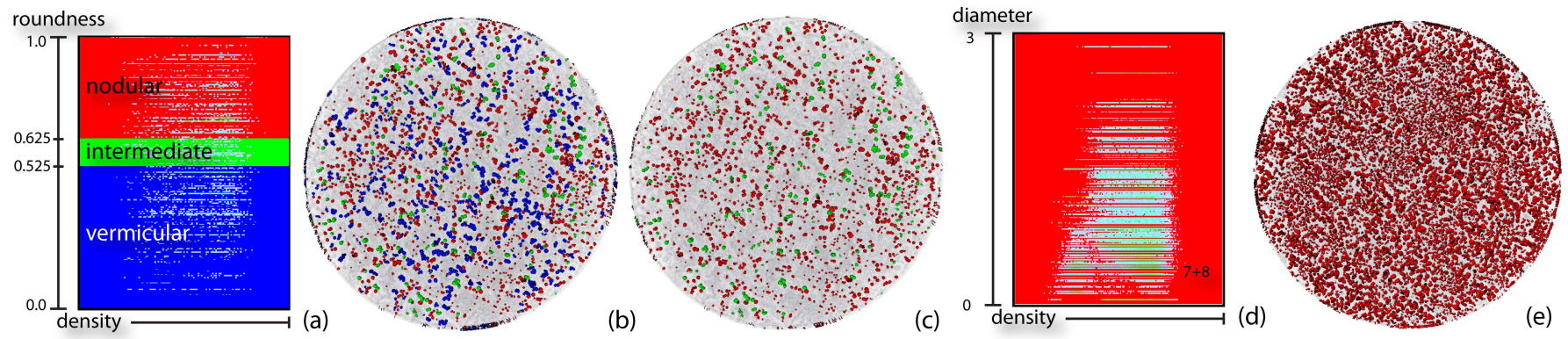

Fig. 9. The same visualization and quantification principle as shown in Figure 8, but for one selected 2D slice, taken from the middle of the volume. Here, the exploration and quantification is based on the same values, but calculated for the 2D case.

shown in Figures 8(d) and (e). With this TF, the user can explore and quantify the different size classes defined in the ISO 945-1 standard. The size on the vertical axis ranges from 0 (class 8 ) to $>100$ (class 1$)$.

\subsection{Discussion and Results}

The established standard workflow is currently based on determining the classification according to the ISO standards in microscopic 2D slice images such as the one shown in Figure 7(a). Due to the significant amount of manual work this entails, all properties are only determined in chosen 2D reference regions, for example the highlighted rectangle in Figure 7(a). This has huge implications for the accuracy of the obtained results. First, the properties under investigation can change considerably from one region in the volume to another one. Second, properties are only determined in 2D cross-sections, i.e., completely independent from the actual 3D shape or volume of the contained particles. Therefore, efforts are underway to move the whole process to $3 \mathrm{D}$ and leverage the possibilities provided by $3 \mathrm{D} \mathrm{CT}$ acquisition. The advantages of extending the quantification to the $3 \mathrm{D}$ domain were discussed in Section 4.1. We have compared the results obtained via our system with results obtained manually for the red box in the 2D slice image in Figure 7(a). Table 4 gives the quantification results for the evaluated parts shown in Figure 8 and Figure 9. The results for $2 \mathrm{D}$ data are comparable considering that differences due to different reference regions used for inspection are to be expected. Furthermore, the different resolutions when comparing microscopic slice images with CT slice images have to be taken into account. Therefore, we have also compared the results obtained by segmenting the corresponding CT slice image with VGStudio MAX shown in Figure 7(b). Comparing with our $2 \mathrm{D}$ results, we see that we have similar results concerning the nodularity, as well as the nodule count. The smaller nodule count emerges from the fact that VGStudio MAX did not segment the small particles, which were taken into account with our system. It was not possible to compare the properties obtained using our system in 3D, since there are no existing reference data. However, as explained above, the results of quantification in $2 \mathrm{D}$ and $3 \mathrm{D}$ are not directly comparable in general. A manual quantification of the $3 \mathrm{D}$ structures in the data to accurately evaluate our system is in progress. Our results show that using the coefficients for form classification de- fined in ISO 16112 for the 2D case have to be exhaustively reviewed when applying this procedure to a potentially much more accurate $3 \mathrm{D}$ application scenario. However, such a review can only be done using a bigger database and is therefore an ongoing process.

Furthermore, graphite inclusions with a diameter less than three voxels must not be included in the analysis to avoid the segmentation of noise. Also shrinkage porosity, which is in most cases much bigger than the graphite particles, is not relevant. Therefore, a minimum and maximum feature size can be specified for the region growing process to exclude a priori undesired features from quantification. This is only an option when the user has a-priori knowledge of the data. In our system, these undesired features can also easily be removed interactively using transfer functions. Once the feature information has been calculated in the pre-processing step, the user can quantify the sample under investigation at any desired slice position and for the whole $3 \mathrm{D}$ volume in real-time. This overcomes the problems mentioned in Section 4.1, which occur during conventional sample preparation.

\section{General Discussion}

In the case of SFRSpC (Section 3), our approach is much faster and reduces the required labor time considerably, since the previous method was almost completely manual. The manual quantification process takes hours for obtaining the same results which we have achieved, even when the short pre-processing times given in Table 6 are considered. For the ductile cast iron (Section 4), our quantification process takes more time than the manual 2D approach when CT acquisition time is also considered and only one 2D slice is prepared manually. However, high quality results can also only be achieved when multiple 2D sections are prepared, which requires an additional 20 minutes per section. Table 5 compares times of the manual and our automatic approach. Additionally, our system provides many powerful possibilities for real-time exploration that enable domain experts to gain more insight about a given material sample. Currently, our system for automatic quantification according to the ISO 945-1 and ISO 16112 standards in $3 \mathrm{D}$ is only used for research purposes. It is a first step towards specific quantification tasks in the 3D domain, but further research and refinement has to be performed in the future until the system can be incorporated into the daily quantification process of metal- 


\begin{tabular}{|c|l|r|r||l|r||r|c|}
\hline application & form & $\%$ & feature count & size & feature count & \% nodularity & nodule count \\
\hline Ductile Cast Iron & VI: & 8.67 & 1349 & $3:$ & 504 & & \\
automatic, 3D & V: & 7.44 & 1268 & $4:$ & 2446 & 12.41 & $1653 \mathrm{~mm}^{-3}$ \\
Fig. 8 (b,c and e) & III: & 83.87 & 9518 & $5:$ & 7079 & & \\
& & & & $6:$ & 2064 & & \\
\hline Ductile Cast Iron & VI: & 45.30 & 1485 & & & & $102 \mathrm{~mm}^{-2}$ \\
automatic, 2D & V: & 15.23 & 286 & $7+8:$ & 2852 & 52.91 & \\
Fig. 9 (b,c and e) & III: & 39.47 & 799 & & & & \\
\hline Ductile Cast Iron & VI: & 46.15 & n/a & & & & \\
manual, VGMax, 2D & V: & 23.08 & n/a & $7+8:$ & 1553 & 53.70 & \\
Fig. 7 (b) & III: & 30.08 & n/a & & & & \\
\hline Ductile Cast Iron & VI: & $>90$ & n/a & & n/a & & \\
manual, microscopic, 2D & V: & $<10$ & n/a & 6 & n/a & 74.80 & $173 \mathrm{~mm}^{-2}$ \\
Fig. 7 (a) & III: & traces & n/a & & n/a & & \\
\hline
\end{tabular}

Table 4. Comparison of the quantification results from the manual 2D approach using the microscopic 2D slice image (a) and the CT data slice image segmented with VGStudio MAX (b) in Figure 7, and the 2D and 3D results of our system, shown in Figure 8 and Figure 9.

lographers. Table 6 gives typical numbers for pre-computation times, memory consumption, and volume rendering frame rates for the data sets used in this paper. Performance has been measured on an Intel Core i7 2.95GHz and an NVIDIA GeForce 285 GTX.

\section{CONCLUSIONS AND FUture Work}

We have presented interactive exploration and quantification of features for two real-world applications of high practical relevance in the building industry. We have demonstrated that our system helps to bridge the gap between visualization, feature detection and quantification, and has the potential of significantly improving the current workflow of NDT practitioners. In the future we would like to integrate more possibilities for interactive measurement, as well as performing further evaluations of correctness of the resulting quantifications. This can be accomplished by further comparisons of manual quantifications, as intended for the 3D case of the graphite microstructure in ductile cast iron. The integration of sub-voxel classification to improve quantification accuracy is also an important future goal.

\section{ACKNOWLEDGMENTS}

We thank the anonymous reviewers, Thomas Höllt, Johanna Beyer, Daniel Habe, Jördis Rosc, Christoph Blasch, Helmut Doleisch, Stephan Mantler, and Vienna Science and Technology Fund WWTF.

\section{REFERENCES}

[1] R. Adams and L. Bischof. Seeded region growing. IEEE Trans. Pattern Anal. Mach. Intell., 16(6):641-647, 1994.

[2] J. Beyer, M. Hadwiger, T. Möller, and L. Fritz. Smooth mixed-resolution gpu-based raycasting. In IEEE/EG Symposium on Volume and PointBased Graphics 2008, pages 163-170, 2008.

[3] C. Blasch. Orientierung von Stahlfasern im Stahlfaserspritzbeton. Bachelor thesis, University of Leoben, 2007.

[4] M. F. Cohen, J. Painter, M. Mehta, and K.-L. Ma. Volume seedlings. In Proc. ACM Symp. on Interactive 3D Graphics, pages 139-145, 1992.

[5] C. Correa and K.-L. Ma. Size-based transfer functions: A new volume exploration technique. IEEE Transactions on Visualization and Computer Graphics, 14(6):1380-1387, 2008.

\begin{tabular}{|c|c|c|}
\hline$\sim$ time for.. & manual 2D & automatic 3D \\
\hline pre-processing & $60 \mathrm{~min}$ (section preparation) & $90 \mathrm{~min}(\mathrm{CT}$ scan $)$ \\
further sections & $20 \mathrm{~min}$ & - \\
analysis & $10 \mathrm{~min}$ & $10 \mathrm{~min}$ \\
\hline & $60+10 \mathrm{~min}+20^{*} \mathrm{x}$ min & $(90+) 10 \mathrm{~min}$ \\
\hline
\end{tabular}

Table 5. Time table comparing the conventional manual approach with our automatic method for quantification of ductile cast iron.

\begin{tabular}{|c|c|c|c|}
\hline data set & resolution & pre-computation & rendering \\
\hline SFRSpC 1 & $440 \times 440 \times 705$ & $1.7 \mathrm{~min}$ & $20-33 \mathrm{fps}$ \\
SFRSpC 2 & $400 \times 400 \times 570$ & $1.2 \mathrm{~min}$ & $25-33 \mathrm{fps}$ \\
Ductile Cast Iron & $932 \times 932 \times 128$ & $21.8 \mathrm{~min}$ & $37-69 \mathrm{fps}$ \\
\hline
\end{tabular}

Table 6. The data sets from this paper, with typical pre-computation times and typical volume rendering frame rates (viewport 512x512).
[6] S. Gottschalk, M. C. Lin, and D. Manocha. Obbtree: A hierarchical structure for rapid interference detection. Computer Graphics, 30(Annual Conference Series):171-180, 1996.

[7] A. Gyulassy, M. Duchaineau, V. Natarajan, V. Pascucci, E. Bringa, A. Higginbotham, and B. Hamann. Topologically clean distance fields. IEEE Transactions on Visualization and Computer Graphics, 13(6):1432-1439, 2007.

[8] M. Hadwiger, L. Fritz, C. Rezk-Salama, T. Höllt, G. Geier, and T. Pabel. Interactive volume exploration for feature detection and quantification in industrial CT data. IEEE Transactions on Visualization and Computer Graphics, 14(6):1507-1514, 2008.

[9] R. Huang and K.-L. Ma. RGVis: Region growing based techniques for volume visualization. Computer Graphics and Applications, Pacific Conference on, 0:355-363, 2003.

[10] R. Huang, K.-L. Ma, P. McCormick, and W. Ward. Visualizing industrial CT volume data for nondestructive testing applications. In Proceedings IEEE Visualization 2003, pages 547-554, 2003.

[11] H. Kerber, G. Schindelbacher, G. Ruess, A. Kneissl, and K. Kutschej. Microstructure characterization of spheroidal graphite cast iron by using various image analytical systems. Practical Metallography 40 (7), pages 335-342, 2003.

[12] J. Kniss, G. Kindlmann, and C. Hansen. Interactive volume rendering using multi-dimensional transfer functions and direct manipulation widgets. In Proceedings IEEE Visualization 2001, pages 255-262, 2001.

[13] J. Krüger and R. Westermann. Acceleration techniques for GPU-based volume rendering. In Proceedings IEEE Visualization 2003, pages 287292, 2003.

[14] MAVI. MAVI - Modular Algorithms for Volume Images, 2009. http: //www. itwm.fhg.de/bv/projects/MAVI/index\_en.php.

[15] H. Schorn. Classification of steel fibre reinforced shotcretes according to recent european standards. In Bernard, E.S. (Hg.): Shotcrete: Engineering Developments, pages 225-229. Swets \& Zeitlinger, 2001.

[16] A. Scozzafava, L. Tomesani, and A. Zucchelli. Image analysis automation of spheroidal cast iron. Journal of Materials Processing Technology, 153-154:853-859, 2004.

[17] H. Singh, A. Gokhale, Y. Mao, and A. Tewari. Reconstruction, visualization, and quantitative characterization of multi-phase three-dimensional microstructures of cast aluminum alloys. In TMS Shape Casting Symposium, pages 15-19, 2009.

[18] S. Stegmaier, M. Strengert, T. Klein, and T. Ertl. A simple and flexible volume rendering framework for graphics-hardware-based raycasting. In Volume Graphics, pages 187-195, 2005.

[19] I. W. Steller, W. Stets, J. Ohser, and D. Hartmann. Computer-aided graphite classification: An approach for international standardization. Trans. of the American Foundry Society Vol. 113, pages 587-594, 2005.

[20] A. Velichko, C. Holzapfel, A. Siefers, K. Schladitz, and F. Mücklich. Unambiguous classification of complex microstructures by their threedimensional parameters applied to graphite in cast iron. Acta Materialia, 56:1981-1990, 2008

[21] VGStudioMAX. Volume Graphics-VGStudio MAX, 2009. http:// www. volumegraphics.com.

[22] S. Zucker. Region growing: Childhood and adolescence. Computer Graphics and Image Processing, 5:382-399, 1976. 